\title{
The Unexpected Kinematics of Multiple Populations in NGC 6362: Do Binaries Play a Role?*
}

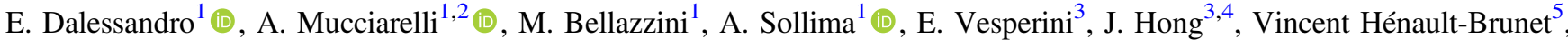 \\ F. R. Ferraro ${ }^{1,2}$ (D), R. Ibata ${ }^{6}$ (D), B. Lanzoni ${ }^{1,2}$ (D), D. Massari ${ }^{7}$ (D), and M. Salaris ${ }^{8}$ \\ ${ }^{1}$ INAF-Astrophysics and Space Science Observatory, Via Gobetti 93/3, I-40129 Bologna, Italy; emanuele.dalessandro@ oabo.inaf.it \\ ${ }^{2}$ Dipartimento di Fisica \& Astronomia, Universitá degli Studi di Bologna, Via Gobetti 93/2, I-40129 Bologna, Italy \\ ${ }^{3}$ Department of Astronomy, Indiana University, Swain West, 727 E. 3rd Street, Bloomington, IN 47405 USA \\ ${ }^{4}$ Kavli Institute for Astronomy and Astrophysics, Peking University, Yi He Yuan Lu 5, HaiDian District, Beijing 100871, People's Republic of China \\ ${ }_{5}^{5}$ National Research Council, Herzberg Astronomy \& Astrophysics, 5071 West Saanich Road, Victoria, BC V9E 2E7, Canada \\ ${ }^{6}$ Observatoire Astronomique, Université de Strasbourg, CNRS, 11 rue de l'Université, F-67000 Strasbourg, France \\ ${ }^{7}$ Kapteyn Astronomical Institute, University of Groningen, Groningen, The Netherlands Leiden Observatory, Leiden University, Leiden, The Netherlands \\ Astrophysics Research Institute, Liverpool John Moores University, IC2 Liverpool Science Park, 146 Brownlow Hill, Liverpool L3 5RF, UK \\ Received 2018 March 29; revised 2018 July 11; accepted 2018 July 17; published 2018 August 29
}

\begin{abstract}
We present a detailed analysis of the kinematic properties of the multiple populations (MPs) in the low-mass Galactic globular cluster (GC) NGC 6362 based on a sample of about 500 member stars for which radial velocities (RVs), and $\mathrm{Fe}$ and $\mathrm{Na}$ abundances have been homogeneously derived. At distances from the cluster center larger than about $0.5 r_{h}$, we find that first-generation (FG-Na-poor) and second-generation (SG-Na-rich) stars show hints of different line-of-sight velocity dispersion profiles, with FG stars being dynamically hotter. This is the first time that differences in the velocity dispersion of MPs are detected using only RVs. While kinematic differences between MPs in GCs are usually described in terms of anisotropy differences driven by the different radial distributions, this explanation hardly seems viable for NGC 6362, where SG and FG stars are spatially mixed. We demonstrate that the observed difference in the velocity dispersion profiles can be accounted for by the effect of binary stars. In fact, thanks to our multi-epoch RV measurements, we find that the binary fraction is significantly larger in the FG sample $(f \sim 14 \%)$ than in the SG population $(f<1 \%)$, and we show that such a difference can inflate the velocity dispersion of FG with respect to SG by the observed amount in the relevant radial range. Our results nicely match the predictions of state-of-the art $N$-body simulations of the co-evolution of MPs in GCs that include the effects of binaries.
\end{abstract}

Key words: globular clusters: general - globular clusters: individual (NGC 6362) - stars: abundances - stars: kinematics and dynamics

\section{Introduction}

The discovery of multiple populations (MPs) in globular clusters (GCs) that differ in light-element abundance (e.g., He, $\mathrm{C}, \mathrm{N}, \mathrm{O}, \mathrm{Na}, \mathrm{Mg}, \mathrm{Al}$ ) while having the same iron (and ironpeak elements) content, has seriously challenged our understanding of the physical mechanisms driving the formation and early evolution of these systems (see Gratton et al. 2012; Bastian \& Lardo 2017 for a review). Indeed, it is now wellestablished that almost all relatively massive $\left(\sim 10^{4} M_{\odot}\right.$; e.g., Piotto et al. 2015; Bragaglia et al. 2017) and old ( $>2$ Gyr; see, for example, Martocchia et al. 2018) GCs host MPs.

Spectroscopically MPs manifest themselves in the form of light-element anti-correlations (like $\mathrm{C}-\mathrm{N}, \mathrm{Na}-\mathrm{O}, \mathrm{Mg}-\mathrm{Al}$ ). These chemical inhomogeneities also produce a variety of features in the color-magnitude diagrams (CMDs) when appropriate near-UV bands are used (Sbordone et al. 2011). Thanks to spectrophotometric studies, MPs have been directly observed in many GCs in the Galaxy (see Piotto et al. 2015 for a recent homogeneous collection), as well as in external systems (like the Magellanic Clouds and the Fornax dwarf galaxy-Mucciarelli et al. 2009; Larsen et al. 2014; Dalessandro et al. 2016). Moreover, the presence of MPs has been indirectly

\footnotetext{
* Based on data obtained with the Very Large Telescope at the European Southern Observatory, programs: 093.D-0618 and 097.D-0325 (PI: Dalessandro).
}

constrained in the GC systems of M31 and M87 (Chung et al. 2011; Schiavon et al. 2013).

Different scenarios have been proposed over the years to explain the formation of MPs. They generally invoke a selfenrichment process, which likely occurred in the very early epochs of GC formation and evolution. In these scenarios, it is thought that a second-generation/population (SG) formed from the ejecta of stars (polluters) of a first-generation/population (FG) mixed with "pristine material" (Decressin et al. 2007; D'Ercole et al. 2008; de Mink et al. 2009; Bastian et al. 2013; Denissenkov \& Hartwick 2014). However, all models proposed so far face serious problems and a self-consistent explanation of the physical processes at the basis of MP formation is still lacking.

Understanding the kinematical properties of MPs can provide new insights into GC formation and evolution. One of the predictions of MP formation models (e.g., D'Ercole et al. 2010) is that SG stars form a low-mass and centrally segregated stellar sub-systems possibly characterized by a more rapid internal rotation than the (more spatially extended) FG system (Bekki 2010). Although the long-term dynamical evolution of stars can smooth out the initial structural and kinematical differences between FG and SG to a large extent, some are expected to still be visible in present-day GCs (see, for example, Vesperini et al. 2013; Hénault-Brunet et al. 2015). The first evidence of the differences in the structural properties of MPs was based on their spatial distributions (Lardo et al. 2011; 
Milone et al. 2012; Bellini et al. 2013; Dalessandro et al. 2016; Massari et al. 2016). These works have shown that indeed SGs are typically more centrally concentrated than FG subpopulations, with few remarkable exceptions (see Dalessandro et al. 2014; Larsen et al. 2015; Dalessandro et al. 2018; Savino et al. 2018). However, spatial distributions alone provide only a partial picture of the dynamical properties of MPs and key constraints on the possible formation and dynamical paths of MPs may be hidden in their kinematic properties.

Hints of different degrees of orbital anisotropy among MPs have been found in the massive GCs 47 Tuc and NGC 2808 by means of HST proper motions (Richer et al. 2013; Bellini et al. 2015). These findings seem to be consistent with the kinematical fingerprints of the diffusion of the SG population from the innermost regions toward the cluster outer zones and provide indirect support to formation scenarios predicting SG formed in a centrally concentrated subsystem.

Cordero et al. (2017) also found that the extremely enriched component of SG stars in M13 shows a larger rotation amplitude than other stars in the cluster.

In general, however, MP kinematics is still poorly constrained, mainly because of technical limitations related to the difficulty of deriving kinematical information in dense environments for large and significant samples of resolved stars adequately separated in terms of their light-element abundances.

Here, we present the results of an extended kinematical analysis of the MPs in the Galactic GC NGC 6362, based on the radial velocities (RVs) of a large sample $(\sim 800)$ of stars chemically tagged according to the subpopulation they belong to. The case of NGC 6362 is particularly interesting. Indeed, this is possibly the second least massive $\left(M \sim 5 \times 10^{4} M_{\odot}\right)$ $\mathrm{GC}^{9}$ where MPs have been identified both photometrically and spectroscopically (Dalessandro et al. 2014; Mucciarelli et al. 2016) so far. Also, contrary to what is generally observed in other GCs (see, e.g., Lardo et al. 2011), we have found that in this cluster the spatial distributions of MPs are consistent with complete mixing over the entire cluster extension (Dalessandro et al. 2014). This behavior suggests that the cluster underwent complete spatial redistribution of stars and severe mass-loss due to long-term dynamical evolution (Vesperini et al. 2013; Dalessandro et al. 2014; Miholics et al. 2015).

The paper is structured as follows. In Section 2, the database and data reduction are presented, in Section 3 sample selection criteria are described, and in Section 4 we report on the main results of the kinematic analysis. In Section 5, subpopulation binary fractions are derived and their impacts are estimated by means of an analytic approach. In Section 6, observations are compared to $N$-body models following the evolution of MPs. Conclusions and a discussion are presented in Section 7.

\section{Observations and Data Analysis}

\subsection{RVs and Chemical Abundances}

The data set used in this work consists of spectra obtained using the multi-object facility FLAMES@ESO-VLT (Pasquini et al. 2000) in the UVES+GIRAFFE combined mode and secured in two observing runs. In the first run (Prop. ID: 093.D0618, PI: Dalessandro), stars were observed using the GIRAFFE setups HR11 and HR13, sampling the two Na

\footnotetext{
9 After NGC 6535, which has $M \sim 2 \times 10^{4} M_{\odot}$; (Piotto et al. 2015; Bragaglia et al. 2017).
}

doublets at 5682-5688 $\AA$ and 6154-6160 $\mathrm{A}$. In the second run (Prop. ID: 097.D-0325, PI: Dalessandro), which was devoted to enlarge the sample of available RVs and $[\mathrm{Na} / \mathrm{Fe}]$ abundances toward fainter magnitudes, we adopted the GIRAFFE setup HR12, in order to cover the Na doublet at 5895-5890 ̊ so that was strong enough to be easily measured at low signal-to-noise ratios (S/Ns). All the UVES targets of both runs have been observed with the UVES Red Arm CD\#3 580 setup. Only red giant branch (RGB) stars brighter than $V \sim 17.6$ and red horizontal branch stars have been selected using the optical and ultraviolet ground-based photometry by Dalessandro et al. (2014). The first run resulted in 219 stars being observed, the second run resulted in 585 stars being observed, and 84 of these stars were shared between the two runs, thus yielding a total of 720 observed stars in the two observing programs. Many stars in the final sample have been observed up to six times. All the spectra have been reduced with the dedicated ESO pipelines. Some results obtained with the first run have been presented in Mucciarelli et al. (2016) and Massari et al. (2017).

RVs have been measured for each individual spectrum with the code DAOSPEC (Stetson \& Pancino 2008) using tens of metallic lines. Heliocentric corrections have been applied to each exposure, then the spectra of each target have been coadded together and used for the chemical analysis. In the analysis presented below we adopt for each star the RV estimate obtained from the spectrum with the highest $\mathrm{S} / \mathrm{N}$. This allows us to homogeneously incorporate the large number of stars with just one spectrum (156) with those with two or more spectra.

Comparing the distribution of the standard deviations $\left(\sigma_{\mathrm{RV}}\right)$ as a function of magnitude, for the stars with at least four independent RV estimates with a distribution of errors on individual best- $\mathrm{S} / \mathrm{N}$ measures $\left(\epsilon_{\mathrm{RV}}\right.$, as derived by DAOSPEC), we noted that, at any given magnitude, $\epsilon_{\mathrm{RV}}$ was systematically smaller than $\sigma_{\mathrm{RV}}$ (see Kirby et al. 2015). We found that multiplying $\epsilon_{\mathrm{RV}}$ by 2 lead to a nearly perfect coincidence of the two distributions, hence we decided to apply this rescaling to get more reliable values of $\epsilon_{\mathrm{RV}}$. These rescaled velocity errors have typical values of $\simeq 0.3 \mathrm{~km} \mathrm{~s}^{-1}$ for $V \leqslant 15.0, \simeq 0.8 \mathrm{~km} \mathrm{~s}^{-1}$ for $15.0<V \leqslant 17.0$, and $\simeq 1.2 \mathrm{~km} \mathrm{~s}^{-1}$ for $V>17.0$.

Stellar atmospheric parameters have been determined as in Mucciarelli et al. (2016). Briefly, effective temperatures and surface gravities have been derived from the available photometry; microturbulent velocities have been obtained spectroscopically.

We derived $\mathrm{Fe}$ abundances from the measured equivalent widths of $\sim 20-30$ (for RGB stars) and $\sim 7-10$ (for HB stars) unblended neutral lines using the code GALA (Mucciarelli et al. 2013b).

$\mathrm{Na}$ abundances have been obtained by fitting the observed lines with a grid of synthetic spectra, in order to take into account the damped wings of the available $\mathrm{Na}$ lines. The $[\mathrm{Na} / \mathrm{Fe}]$ abundances have been corrected for departures from local thermodynamical equilibrium according to Lind et al. (2011). For the sake of consistency we also applied the same corrections to the $\mathrm{Na}$ abundances derived in Mucciarelli et al. (2016). Since the $[\mathrm{Na} / \mathrm{Fe}]$ abundances of the two data sets are based on different $\mathrm{Na}$ lines, some differences in the resulting abundances might be expected. By using stars in common between the two data sets, we derived an average shift of $(+0.08 \pm 0.01)$ dex, which was added before combining all the available measures. 

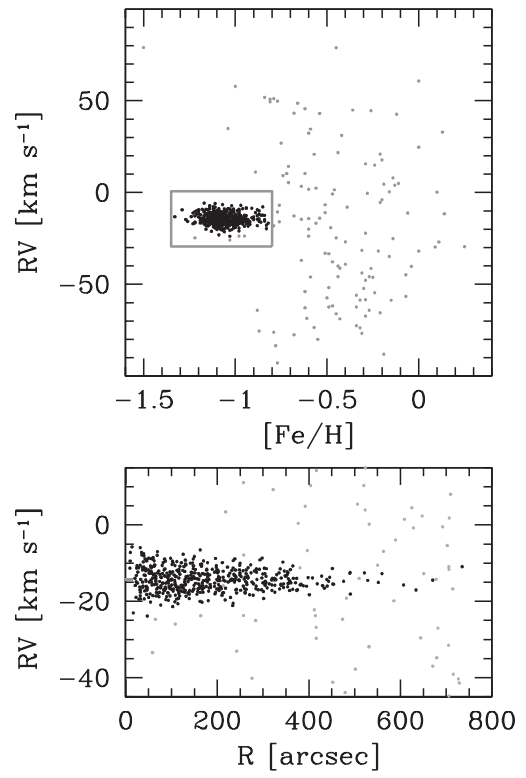

Figure 1. Distribution of RVs of the stars in our sample as a function of the derived $[\mathrm{Fe} / \mathrm{H}]$ (upper panel) and projected distance from the cluster center (bottom panel). The black dots represent stars selected as described in Section 3 and used for the kinematic analysis.

Uncertainties in the abundance ratios have been computed according to the procedure described in Mucciarelli et al. (2013a), which includes both the errors related to the measure of EWs and those arising from the atmospheric parameters.

\section{Sample Selection}

Since our main goal is to compare the kinematic properties of FG and SG stars, we adopted rather strict selection criteria to avoid contamination from spurious signals of any origin. For this reason we excluded from the final sample all the stars (a) lacking reliable $\mathrm{Na}$ abundance estimates (mainly due to low $\mathrm{S} / \mathrm{N}$ or defects in the spectra), (b) with $\epsilon_{\mathrm{RV}}$ anomalously large for their magnitude, namely $\epsilon_{\mathrm{RV}}>0.8 \mathrm{~km} \mathrm{~s}^{-1}$ for $V \leqslant 14.9, \epsilon_{\mathrm{RV}}>$ $1.8 \mathrm{~km} \mathrm{~s}^{-1}$ for $14.9<V \leqslant 16.8$, and $\epsilon_{\mathrm{RV}}>2.8 \mathrm{~km} \mathrm{~s}^{-1}$ for $V>$ 16.8 , and (c) having $\sigma_{\mathrm{RV}}>3.0 \mathrm{~km} \mathrm{~s}^{-1}$, as they are likely binary stars with large velocity amplitude.

Figure 1 shows the distribution of RVs as a function of $[\mathrm{Fe} / \mathrm{H}]$ and as a function of the projected distance from the cluster center $(R)$ for the 632 stars that survived the above selection. Stars belonging to NGC 6362 are easily identified in the $\mathrm{RV}-[\mathrm{Fe} / \mathrm{H}]$ diagram, as they stand out from the field population at $\sim-15 \mathrm{~km} \mathrm{~s}^{-1}$ and $[\mathrm{Fe} / \mathrm{H}] \sim-1.1$. We selected as cluster members the stars within the dark gray box shown in Figure 1 (upper panel), enclosing stars with $-29.3 \mathrm{~km} \mathrm{~s}^{-1} \leqslant$ $\mathrm{RV} \leqslant+0.7 \mathrm{~km} \mathrm{~s}^{-1}$ and $-1.35<[\mathrm{Fe} / \mathrm{H}]<-0.8$. Finally, studying the velocity distribution of member stars as a function of $R$, we identified four stars whose $\mathrm{RVs}$ deviate from the systemic velocity of the cluster by more than three times the local value of the velocity dispersion, i.e., the velocity dispersion in a small radial range about their position. Using these selection criteria, we selected a total of 489 bona fide member stars, which we define here as the total sample, plotted as black dots in Figure 1. Applying the maximum likelihood (ML) algorithm described in Mucciarelli et al. (2012) to the set of 489 individual $[\mathrm{Fe} / \mathrm{H}]$ estimates and errors, we find
$\langle[\mathrm{Fe} / \mathrm{H}]\rangle=-1.063 \pm 0.004$ and $\sigma_{[\mathrm{Fe} / \mathrm{H}]}=0.000 \pm 0.006$, in agreement with Mucciarelli et al. (2016). It is reassuring that the outcome of our selection is a sample drawn from a pure single-metallicity population that is thus very likely composed only of genuine cluster members.

The $\mathrm{Na}$ abundance distribution of member stars (Figure 2 panel (a)) is clearly bimodal. This is in agreement with Mucciarelli et al. (2016) based on the much smaller sample (160 member stars) from the first observing run. Using the Gaussian mixture modeling algorithm described by Muratov \& Gnedin (2010), we find that the hypothesis of unimodal distribution can be rejected with a probability of $>99.9 \%$. Based on the shape of the distribution shown in Figure 2 (panel (a)) we define two subpopulations. The first, here defined as the Na-poor sample, includes stars with $[\mathrm{Na} / \mathrm{Fe}]<+0.05$ and has a total of 288 stars. The second, the Na-rich sample, is composed of stars with $[\mathrm{Na} / \mathrm{Fe}]>+0.05$, for a total of 201 stars. We have checked that the adoption of slightly different boundaries does not affect our results (see Section 4). We verified that the two subsamples have indistinguishable radial distributions consistent with their parent populations. Based on our present understanding of MP formation, in the following we will sometimes refer to the Na-poor and Na-rich subsamples as FG and SG stars, respectively.

Panel (b) of Figure 2 shows the distribution of Na-poor and Na-rich stars in the $(U, U-B)$ CMD (Dalessandro et al. 2014). The two groups appear to be nicely separated on the RGB and in particular, Na-poor stars reside on the bluer side of the RGB (as they are N-poor), while the Na-rich component lies on the red side, as expected (Sbordone et al. 2011).

\section{Kinematic Analysis and Results}

In Figure 3 the line-of-sight velocity dispersion profiles of the two considered subsamples are compared. In the upper panel we show the binned dispersion profiles for illustration purposes only. They are obtained by assuming an equal number of stars in each bin (apart from the outermost bin, which contains any additional leftover stars). We used the ML estimator of Pryor \& Meylan (1993) to compute the velocity dispersion in each bin and its uncertainty. At each bin we assigned the value of the distance corresponding to the mean radius of all the stars in that bin. The corresponding horizontal error bars represent the radial range spanned by the stars in a given bin.

To characterize the kinematics of the Na-rich and Na-poor samples, we used the ML method described in Cordero et al. (2017). We stress that this is a purely kinematic approach aimed at searching for relative differences in the kinematics of subpopulations and not aimed at providing a self-consistent dynamical model of the system. For each subsample, we fit a kinematic model to discrete RVs. As in Cordero et al. (2017), we assume for the velocity dispersion profile the functional form of the Plummer (1911) model, defined by its central velocity dispersion $\sigma_{0}$ and its scale radius $a$ :

$$
\sigma^{2}(R)=\frac{\sigma_{0}^{2}}{\sqrt{1+R^{2} / a^{2}}},
$$

where $R$ is the projected distance from the center of the cluster. Table 1 lists the best-fit values and the uncertainties of the main 

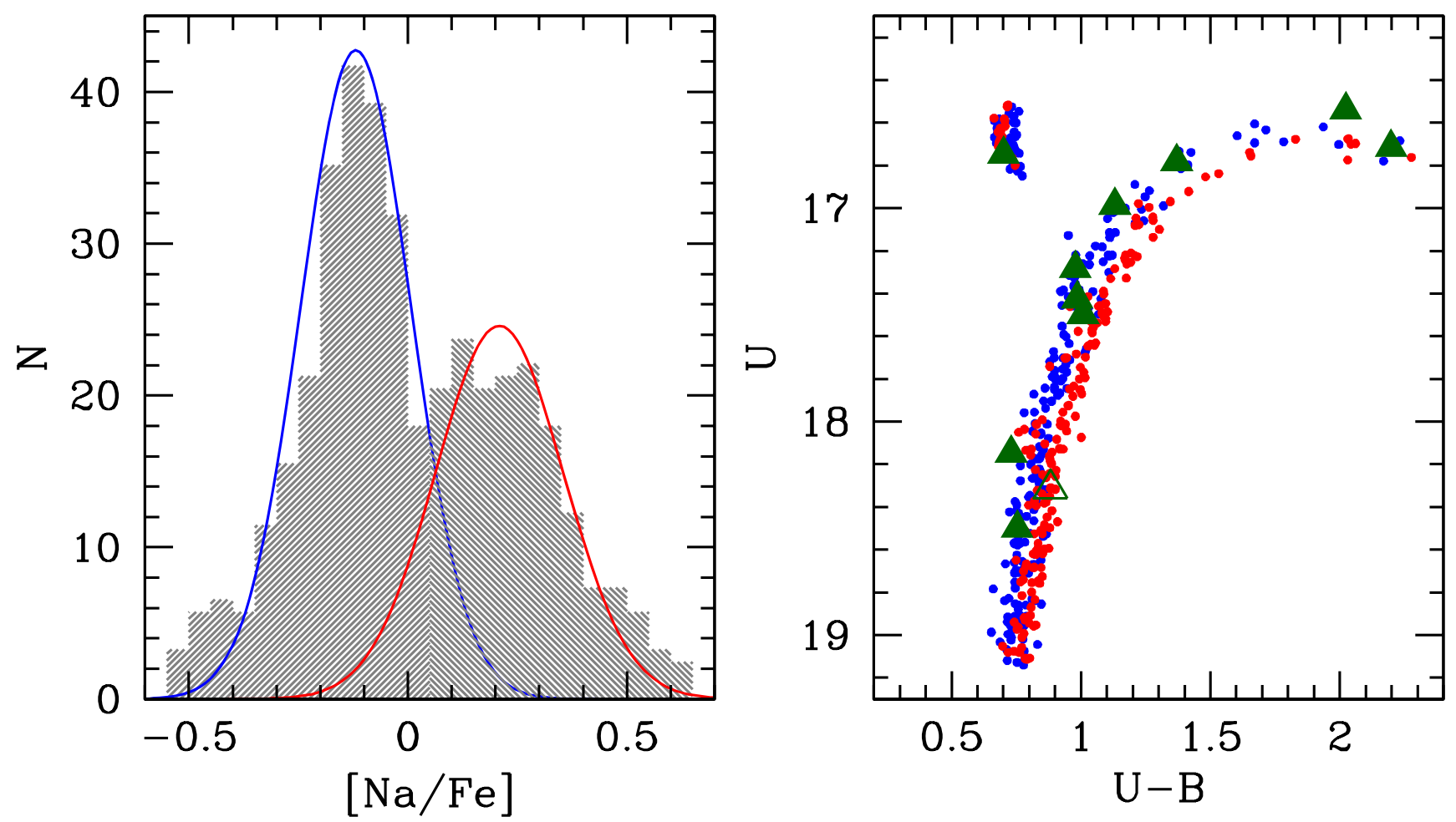

Figure 2. Left panel: distribution of the $[\mathrm{Na} / \mathrm{Fe}]$ abundances of the 489 selected stars. Right panel: $(U, U-B) \mathrm{CMD}$ of the selected samples (photometry from Dalessandro et al. 2014); Na-rich stars are in red and Na-poor ones are in blue. The filled green triangles are Na-poor binary stars directly detected as described in Section 5; the open triangle is a Na-rich binary.

parameters of our analysis and for the different samples considered.

In the bottom panel of Figure 3 the best-fit Plummer models and the 1- $\sigma$ confidence envelopes on the dispersion profiles are also shown.

A comparison of the velocity dispersion profiles, in particular of those obtained without binning the data, clearly shows differences between the velocity dispersion profile of the FG and the SG populations. In particular, while the two profiles are indistinguishable out to about $\sim 70^{\prime \prime}-80^{\prime \prime}$ (corresponding to $\sim 0.5 \times R_{h}$ and $\sim 1.5 R_{c}$; Dalessandro et al. 2014), beyond this radius the SG velocity dispersion profile decreases more sharply than that of the FG and attains values of the dispersion smaller than those of the FG population by $\sim 1 \mathrm{~km} \mathrm{~s}^{-1}$. This difference represents a large fraction $(\sim 30 \%)$ of the observed central velocity dispersion values of Na-poor and Na-rich stars $\left(\sigma_{0} \sim 3.55_{-0.28}^{+0.38} \mathrm{~km} \mathrm{~s}^{-1}\right.$ and $\sigma_{0} \sim 3.61_{-0.48}^{+0.60} \mathrm{~km} \mathrm{~s}^{-1}$, respectively) $)^{10,11}$

We note that this is the first time that differences in the lineof-sight velocity dispersions of MPs are detected.

In order to quantitatively assess the significance of this result, we performed a Kolmogorov-Smirnov test on the RVs. We find that the probability that the two populations are

\footnotetext{
${ }^{10}$ On the other hand, we found no significant difference in the overall rotation pattern of the two subsamples, adopting both the approach of Bellazzini et al. (2012) and that of Cordero et al. (2017). A more detailed analysis of the rotation properties of the cluster will be presented in a companion paper (E. Dalessandro et al. 2018, in preparation).

${ }^{11}$ We point out that we use a Plummer model just as a convenient way of providing a quantitative characterization of kinematical differences; the differences in this kinematical characterization are not meant to have any implications for the spatial distribution of the two populations, which, as shown in Dalessandro et al. (2014), are spatially mixed.
}

Table 1

Median Value and $\pm 1 \sigma$ Uncertainties (Enclosing the Central 68\% of the Probability Distribution) Obtained from the Posterior Probability Distributions for the Free Parameters of the Different Subsamples

\begin{tabular}{lcccc}
\hline \hline Sample & $N_{\text {stars }}$ & $\begin{array}{c}v_{0} \\
\left(\mathrm{~km} \mathrm{~s}^{-1}\right)\end{array}$ & $\begin{array}{c}\sigma_{0} \\
\left(\mathrm{~km} \mathrm{~s}^{-1}\right)\end{array}$ & $\begin{array}{c}a \\
(\operatorname{arcmin})\end{array}$ \\
\hline Na-poor & 288 & $-14.10_{-0.19}^{+0.20}$ & $3.55_{-0.28}^{+0.38}$ & $2.98_{-0.96}^{+1.43}$ \\
Na-rich & 201 & $-14.61_{-0.22}^{+0.22}$ & $3.61_{-0.48}^{+0.60}$ & $1.69_{-0.63}^{+1.11}$ \\
Total sample & 489 & $-14.33_{-0.14}^{+0.14}$ & $3.61_{-0.28}^{+0.32}$ & $2.13_{-0.55}^{+0.76}$ \\
\hline
\end{tabular}

extracted from the same parent distribution is $P_{\mathrm{KS}} \sim 4 \times$ $10^{-3} \cdot{ }^{12}$ We have also performed a $F$-test to test the hypothesis that the velocity dispersions of the two samples are equal. We used an ML analysis to derive the intrinsic velocity dispersions for the two sub-samples at different radial distances from the cluster center. We find that for $R>150^{\prime \prime}$ (which corresponds to the distance where velocity dispersion profiles Na-poor and Na-rich start to differ) the F-test probability is only $\sim 4 \times 10^{-3}$ and it further decreases for $R>200^{\prime \prime}\left(2 \times 10^{-3}\right)$. These results therefore provide strong support for the conclusion that the Napoor and Na-rich velocity dispersion profiles are significantly different, with Na-poor stars being dynamically hotter.

Considering the results of our previous study of MPs in NGC 6362 (Dalessandro et al. 2014) that showed that the spatial distributions of the FG and SG populations are

\footnotetext{
12 This test is not directly addressing the significance of the differences between the velocity dispersion profiles, but of the RV distribution. In this respect it important to note that the shape of the cumulative distributions as well as the derived probabilities also depend on the mean velocities of the two samples.
} 


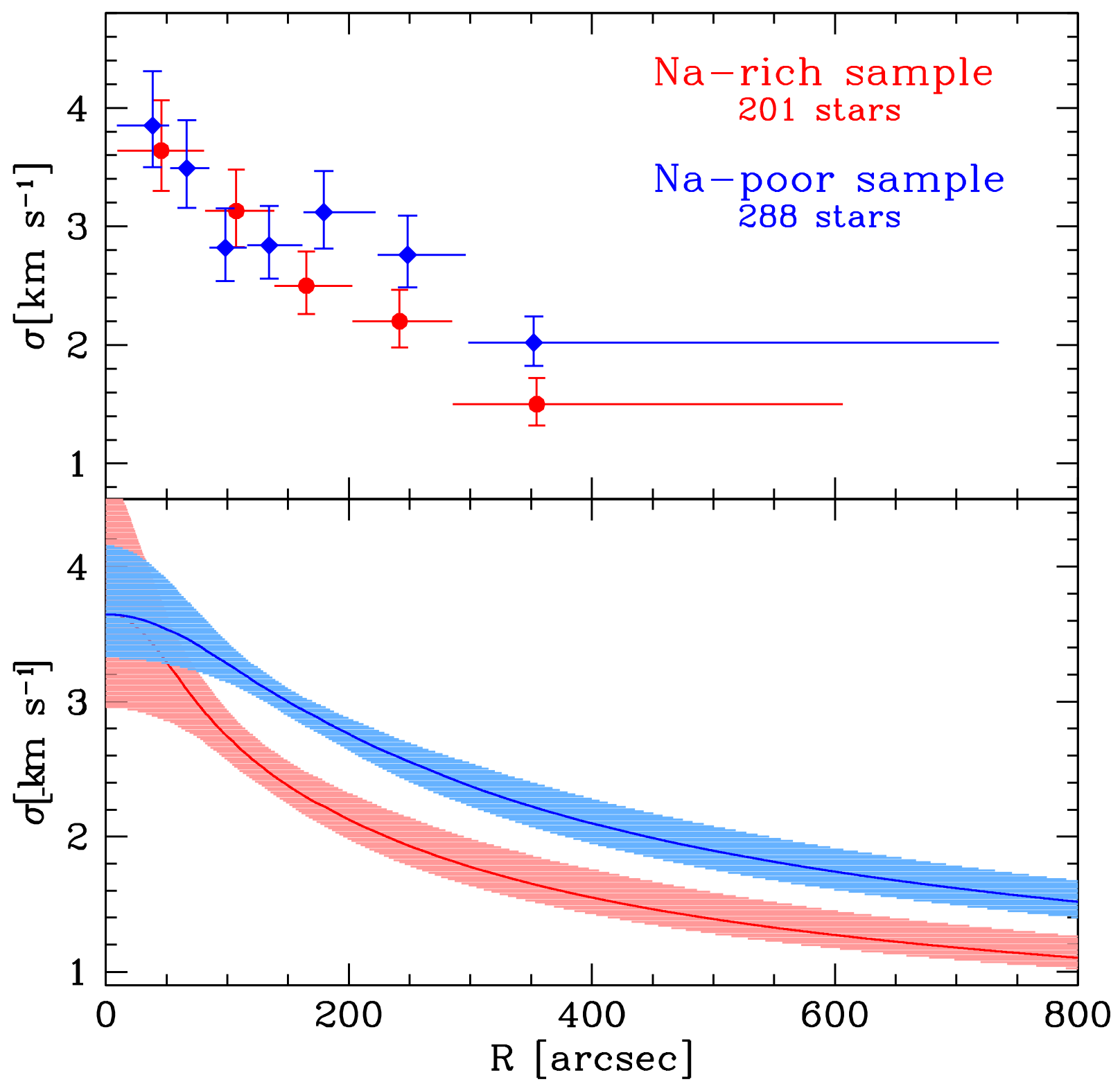

Figure 3. Binned velocity dispersion profiles (upper panel) and $1 \sigma$ confidence regions around the best-fit dispersion profiles (bottom panel) for the Na-rich (red) and Na-poor (blue) samples.

consistent with being completely mixed, the kinematic difference we have found is surprising. Complete spatial mixing is expected in the advanced stages of a cluster's evolution and after the cluster has undergone significant massloss. As we will further discuss in Section 6, once a cluster has attained complete spatial mixing, its MPs should also be characterized by similar velocity dispersion profiles. The results found here therefore raise a fundamental question concerning the dynamical ingredients responsible for the observed kinematic differences.

\section{FG and SG Binary Fractions}

Before exploring the possible culprit of the observed kinematic differences, we use our RV data to determine the binary fraction in the FG and SG populations. Information about the binary population and the possible differences between the FG and SG binary fractions is extremely important both to build a complete dynamical picture of the cluster and to shed light on the possible role of binaries on the observed kinematic properties presented in the previous section. The finding by Lucatello et al. (2015) that FG stars typically have a larger binary fraction than their SG counterparts seems particularly relevant in this context.

We used a subsample of 384 stars observed repeatedly (from a minimum of 2 to a maximum of 6 times; see Section 2) within a period of 704.96 days ( $\sim 2$ years). Candidate binary stars (i.e., those with $\sigma_{\mathrm{RV}}>3 \mathrm{~km} \mathrm{~s}^{-1}$ - see Section 3) have now been included in the analysis. We stress that this is the largest sample ever used so far for this kind of study for an individual GC.

We followed the approach described in Lucatello et al. (2015) for the binary fraction derivation. For each star in our subsample, we performed a $\chi^{2}$ test using the single velocities and relative errors to assess whether they are compatible with a non-variable behavior. Stars with $P\left(\chi^{2}\right)<1 \%$ have been flagged as binaries. Among the 384 stars (235 belonging to the FG and 149 to the SG), 12 turned out to be binaries, 11 are FG stars, and 1 belongs to the SG subpopulation. These values 


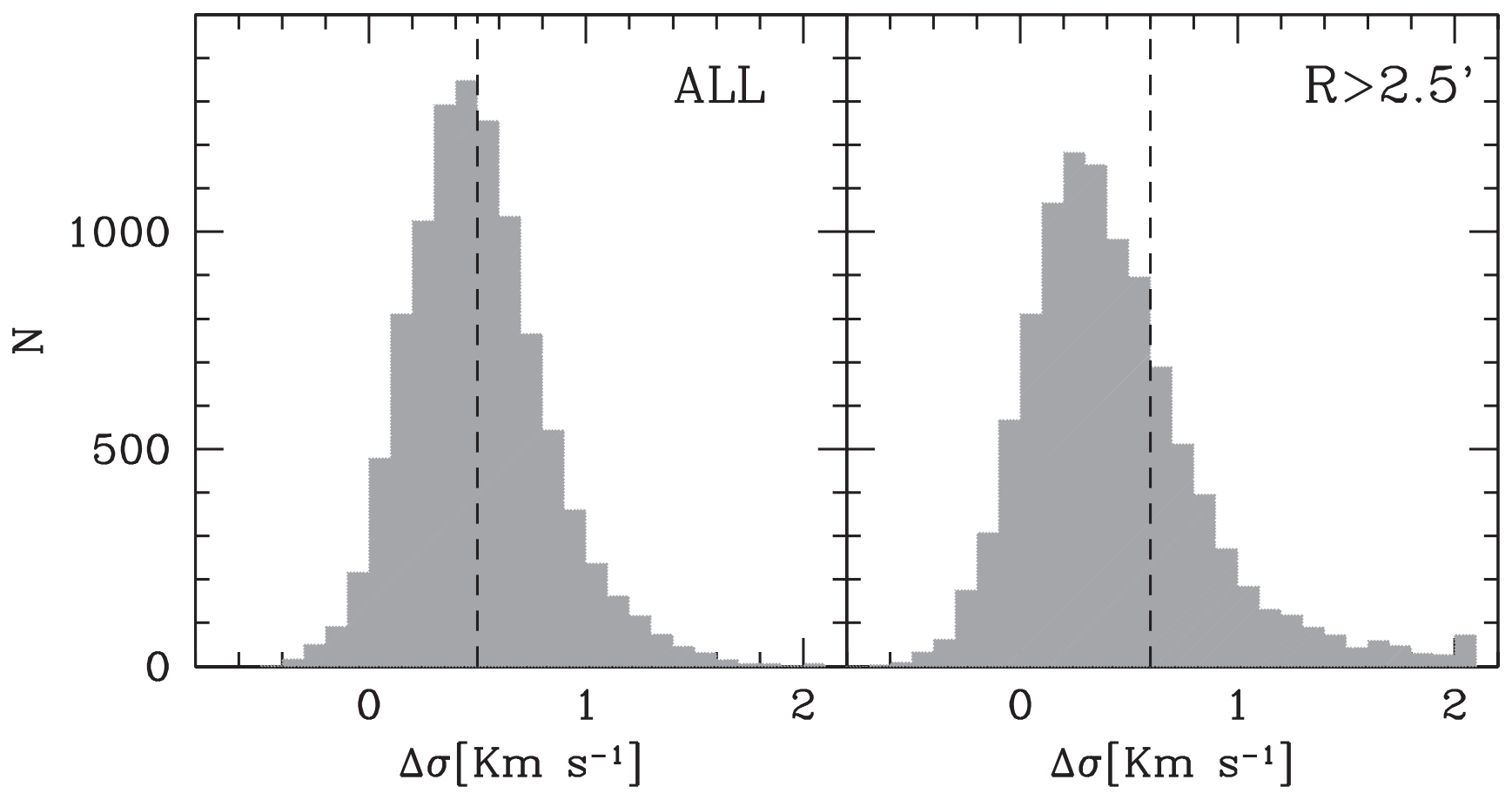

Figure 4. Distributions of the velocity dispersion differences obtained as described in Section 4 for the entire sample and for stars located at $R>2.5$ from the cluster center. The dashed lines represent the observed velocity dispersion differences.

correspond to a minimum binary fraction for the entire sample $(\mathrm{FG}+\mathrm{SG}) f_{\min }^{\mathrm{TOT}}=3.1 \pm 0.9 \%$, and to $f_{\min }^{\mathrm{FG}}=4.7 \pm 1.4 \%$ and $f_{\min }^{\mathrm{SG}}=0.7 \pm 0.7 \%$.

Unfortunately, a significant fraction of binaries cannot be directly detected with our data set because of their long variability periods and/or unfavorable inclination angles. In order to account for these observational limitations and estimate the binary detection efficiency of our observations, we adopted the following approach. For each observed star, we generated a synthetic population of 1000 binaries assuming a primary component mass of $0.85 M_{\odot}$ (adequate for an RGB star in NGC 6362), secondary component masses randomly extracted from a flat mass-ratio distribution, periods extracted from a $\log$-normal distribution (using $\langle\log (P / d)\rangle=4.8$ and $\sigma_{\log (P / d)}=2.3$; Duquennoy \& Mayor 1991), eccentricities extracted using the prescriptions of Duquennoy \& Mayor (1991), random inclination angles, periastron longitude, and orbital phases. We also forced the binary semimajor axes to lie between the Roche-Lobe overflow distance limit (Lee \& Nelson 1988) and that corresponding to the average collisional ionization limit (Hut \& Bahcall 1983). The adoption of this additional criterion reduces the extent of the simulated period distribution to the range $0.4<\log (P / d)<5.3$. For each synthetic binary, the luminosity-weighted systemic velocities have been computed and sampled with the same cadence as the observations.

Gaussian shifts with standard deviations equal to observational errors have been added to the velocities to mimic the effects of observational uncertainties. As a control population, we also simulated a large number of single stars with constant velocity.

The same analysis performed on the observed sample has been applied to the synthetic populations of binaries and single stars to derive the detection efficiency as a function of period and the false detection frequency. The detection efficiency ranges from $\sim 40 \%$ at $\log (P / d)=0.6$, to $\sim 60 \%$ at $\log (P / d)=$ 1.6 , with a decreasing tail at large periods reaching zero at log $(P / d)>4.5$. Overall, an average detection efficiency of $\sim 26 \%$ has been found, while the false detection frequency is $\sim 1 \%$. FG stars have a slightly larger detection efficiency with respect to SG ones (26.7\% versus $19.5 \%)$, likely due to the smaller velocity measurements errors in FG stars characterized by strong $\mathrm{Na}$ lines. Based on these results, the global binary fractions of FG and SG turn out to be $f_{g}^{\mathrm{FG}} \sim 14.3 \%$ and $f_{g}^{\text {SG }}<1 \%$, respectively.

This analysis, which is based on a sample up to 8 times larger (per individual cluster), confirms previous findings (Lucatello et al. 2015) about the different present-day binary fractions of FG and SG subpopulations. It also provides support to the predictions of the theoretical studies of Vesperini et al. (2011) and Hong et al. $(2015,2016)$ that theorized that the SG binary fraction is expected to be smaller than that of the FG population as a result of dynamical evolution.

\subsection{The Effect of Binary Fraction Differences on the Velocity Dispersion Profiles}

To estimate the potential effect of such different binary fractions on the derived velocity dispersion profiles of FG and SG subpopulations, we first adopted the following approach. Mock observations have been constructed by randomly extracting for each observed star a velocity from a Gaussian function with a dispersion equal to the line-of-sight velocity dispersion profile at the same cluster-centric distance of the observed star. The same line-of-sight velocity dispersion profile has been adopted for FG and SG stars, following the King (1966) model that best fits the cluster density profile (see Dalessandro et al. 2014). A random number $(r n)$ comprised in the range $0-1$, extracted from a uniform probability distribution, was assigned to each FG star. For stars with $r n<0.143$, which corresponds to the estimated global FG binary fraction, a 


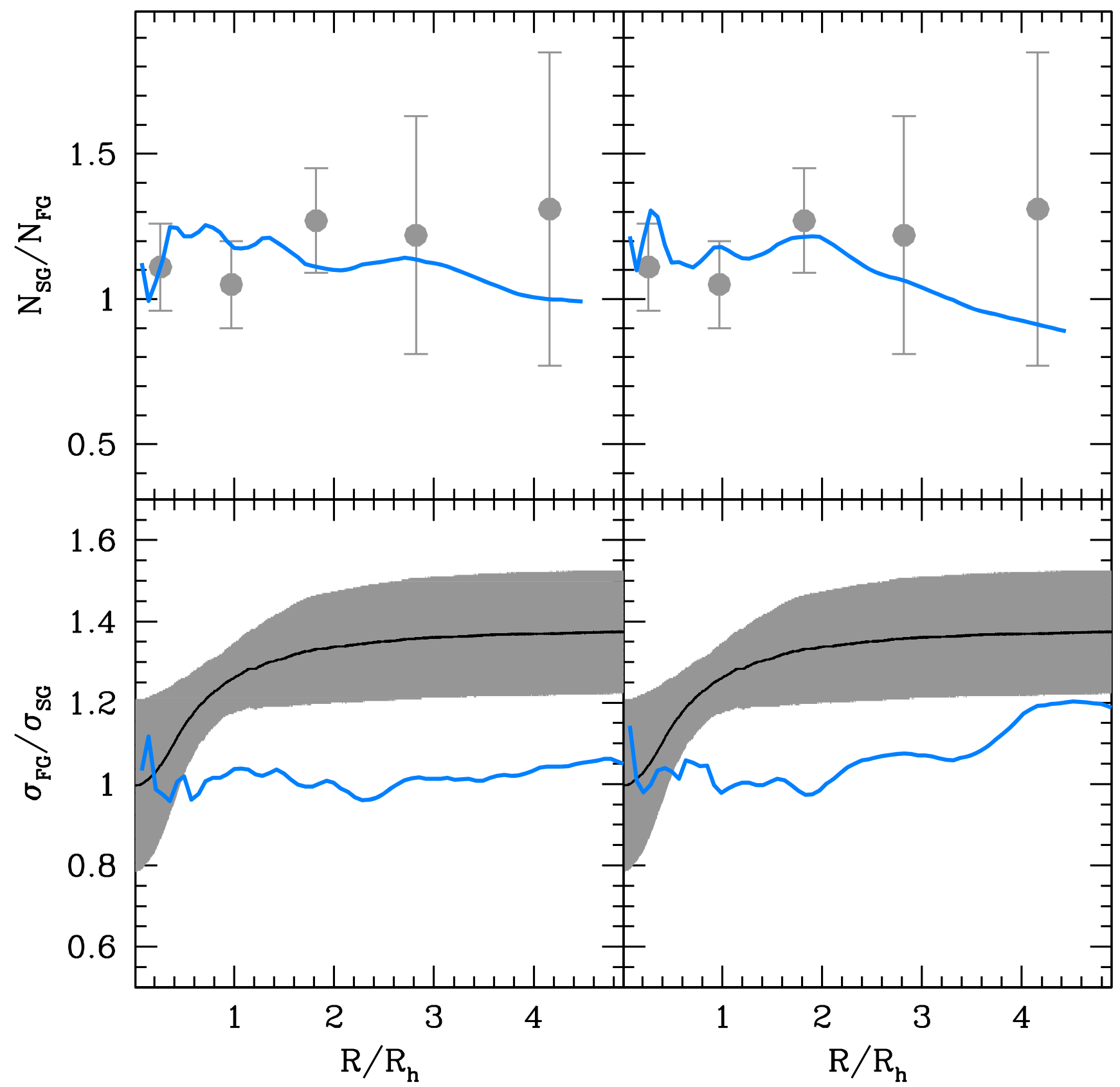

Figure 5. Upper panels: FG and SG number ratio as a function of the distance from the cluster center normalized to $R_{h}$ in two advanced snapshots of our $N$-body simulations with no binaries (blue lines). The gray circles represent the number ratio distribution observed in NGC 6362 (Dalessandro et al. 2014). Lower panels: radial variations of the ratio of the observed FG to SG line-of-sight velocity dispersions $\left(\sigma_{\mathrm{FG}} / \sigma_{\mathrm{SG}}\right)$ from $N$-body models for the same snapshots as before. The dashed gray area represents the observations.

synthetic binary has been simulated with the mass ratios and orbital parameters described above and the corresponding mean velocity shift has been added. The LOS velocity dispersions of the two generations have then been computed using (a) the entire sample and (b) only stars with $R>150^{\prime \prime}$ (i.e., where the maximum difference has been observed). The same selection criteria adopted for the construction of the observed dispersion profiles and to exclude apparent binaries have been applied in the analysis of the mock observations. A set of 1000 extractions has been performed for both the considered radial ranges and the distribution of differences has been derived. The results are shown in Figure 4. It can be seen that a velocity dispersion difference equal or larger than the observed one can be obtained as a result of the effect of the different binary fractions in $\sim 54 \%$ of the simulated cases (if the entire radial range is considered) and in $\sim 28 \%$ of the cases when only the outermost radial range is considered. Hence, the observed difference in the velocity dispersion profile between FG ad SG stars in NGC 6362 is consistent with being due to the difference in the binary fraction of the two populations.

\section{6. $N$-body Models}

In order to further explore the possible dynamical history behind the kinematical differences revealed by our observations, we have studied the evolutions of the line-of-sight velocity dispersions of FG and $\mathrm{SG}$ stars in an $N$-body simulation. The goal here is not to present a model specifically 


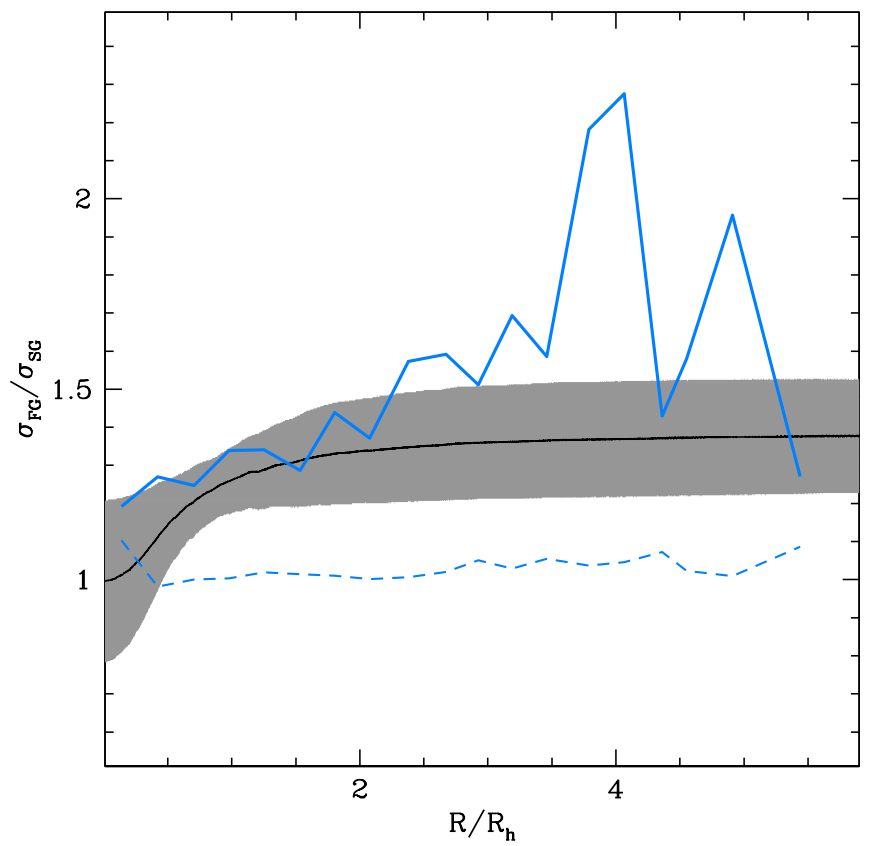

Figure 6. Radial variations of the ratio of the observed FG to SG line-of-sight velocity dispersions $\left(\sigma_{\mathrm{FG}} / \sigma_{\mathrm{SG}}\right)$ from $N$-body models with binaries (see Section 6) compared to observations (gray dashed area). The dashed line represents the velocity dispersion ratio radial distribution from the same simulation when the effect of binaries is not included.

tailored to fit in detail the dynamics of NGC 6362, but rather to gather some fundamental insight into the dynamical ingredients necessary to explain the observational results. For details on the initial conditions of the simulation we refer to Vesperini et al. (2018). Here, we just summarize the main points. Our simulation starts with a SG population embedded within a more extended FG population: the initial half-massradius of the FG population is about five times larger than that of the SG population. The simulation starts with 50,000 stars equally split between FG and SG. In this simulation we have focused our attention on the effects of two-body relaxation on the evolution of the spatial and kinematical properties of the two populations.

In Figure 5 we show the radial profile of the number ratio of SG to FG stars (upper panels) and the ratio profiles of the FG to SG line-of-sight velocity dispersions $\left(\sigma_{\mathrm{FG}} / \sigma_{\mathrm{SG}}\right.$; lower panel) representative of two advanced evolutionary stages in our simulations for stars with masses between 0.75 and $0.85 M_{\odot}$. Although as pointed out above, our analysis is not aimed at providing a detailed fit of the observations, we also include in these plots the observational data from the analysis carried out in this paper and in Dalessandro et al. (2014) to allow a more quantitative comparison between the strengths of the observed and theoretical gradients.

The plots of Figure 5 shed light on the close connection between the structural and kinematical properties of the FG and $\mathrm{SG}$ populations. For a dynamically old cluster in which the FG and the SG populations are completely spatially mixed (Figure 5 left panels), the $N$-body simulations show that the radial profile of $\sigma_{\mathrm{FG}} / \sigma_{\mathrm{SG}}$ is flat, at odds with the observations. If we consider a dynamical phase characterized by a modest radial gradient in the fraction of SG stars (but still consistent within the errors with the observations; Figure 5 right panels), the $\sigma_{\mathrm{FG}} / \sigma_{\mathrm{SG}}$ tends to increase in the external regions of the cluster, but the values of $\sigma_{\mathrm{FG}} / \sigma_{\mathrm{SG}}$ remain smaller than the observed ones at all distances from the cluster center.

Although the discrepancy between the theoretical and the observed radial profile of $\sigma_{\mathrm{FG}} / \sigma_{\mathrm{SG}}$ can be of the order of $\sim 1.5 \sigma$ in the external regions, the systematic underestimate of the ratio compared to the observed one could be an indication that an additional dynamical effect not accounted for in the simulations might be responsible for the observed profile. Indeed, as discussed in Section 5.1, a difference between the FG and SG binary fractions can play a key role in determining the observed differences between the FG and SG velocity dispersion profiles.

As shown in Vesperini et al. (2011) and Hong et al. (2015, 2016), if SG stars form in a more compact and centrally concentrated subsystem than FG stars, as predicted by a number of formation models (see e.g., D'Ercole et al. 2008), all the processes altering the number and orbital properties of binary stars (ionization, hardening, softening, ejection; see, e.g., Heggie \& Hut 2003) affect the SG binaries more efficiently than the FG ones. One of the consequences of this dynamical difference is the preferential disruption and ejection of SG binaries, which in turn leads to a larger global fraction of FG binaries.

As shown in the study of Hong et al. (2016) and J. Hong et al. (2018, in preparation), the processes affecting the evolution and survival of binaries also have an effect on their spatial distributions and the spatial mixing of the FG and SG binaries. In particular, the timescale for the spatial mixing of FG and SG binaries can be much longer than that of single stars. This implies that while the FG and SG single stars might have already reached complete spatial mixing, the FG and SG binary populations might still be characterized by a radial gradient, with the fraction of FG to SG binaries increasing as the cluster-centric radius increases (see, e.g., the bottom panel of Figure 11 in Hong et al. 2016). The difference in the fraction of FG and SG binaries and its radial variation imply that the possible velocity dispersion inflation due to binaries is stronger for the FG population and is increasingly more important at larger distances from the cluster center. As a consequence, differences in the FG and SG binary fractions and in their spatial distributions, can contribute to producing a $\sigma_{\mathrm{FG}} / \sigma_{\mathrm{SG}}$ profile that increases with distance from the cluster center, as found in our observations.

Figure 6 illustrates this effect as measured in one of the simulations (MPr5f1x3-800) presented in Hong et al. (2016). We emphasize that the simulations are still idealized and are not meant to provide a detailed model for NGC 6362, but they include the essential dynamical ingredients necessary to illustrate the effect of interest here. From this analysis and the comparison with the observations, it emerges that binaries can play a major role in shaping the $\sigma_{\mathrm{FG}} / \sigma_{\mathrm{SG}}$ radial gradient and could be the dynamical ingredient needed to match the observational results.

Although additional and more realistic simulations are needed to build specific models for NGC 6362, the results presented here clearly illustrate how the study of the kinematics of MPs can reveal the fingerprints of a number of fundamental dynamical effects and of their role in shaping the properties of FG and SG stars.

\section{Summary and Discussion}

The detailed kinematic analysis performed in this work has revealed that Na-poor (FG) and Na-rich (SG) stars are characterized by significantly different line-of-sight velocity 
dispersion profiles. SG stars have systematically smaller velocity dispersion values than FG ones, with differences of $\sim 1 \mathrm{~km} \mathrm{~s}^{-1}$ for $R>70^{\prime \prime}-80^{\prime \prime}$ (corresponding to $R>0.5 R_{\mathrm{h}}$ ). This is the first time that differences in the line-of-sight velocity dispersion of MPs are detected.

Considering that in our previous study on the spatial distribution of MPs in NGC 6362 (Dalessandro et al. 2014), we have found that the FG and SG populations are spatially mixed and that the cluster must be in an advanced stage of its dynamical evolution, the kinematical evidence detected here is surprising and raises a fundamental question concerning the dynamical processes responsible for the difference between the FG and SG velocity dispersion profiles.

Thanks to our large set of RVs, we have also been able to estimate the binary fraction in the two populations and found a significant difference between the FG binary fraction $(f \sim 14 \%)$ and that of the SG population $(<1 \%)$. This result is based on the largest sample ever used for this kind of analysis.

By using $N$-body simulations and mock observations, we show that such a large binary fraction difference can play an essential role in determining the observed kinematic differences between the FG and the SG populations found in our study.

Besides the specific case of NGC 6362, the results of this paper clearly demonstrate the importance of the study of the kinematics at several epochs to build a complete dynamical picture of MPs in GCs and to shed light on the dynamical history of MPs. In this context it will be important to extend this kind of analysis to other systems in order to understand whether NGC 6362 is a peculiar case or similar effects are present in all GCs. Moreover, the addition of Gaia proper motions sampling the entire extension of the cluster will allow us to constrain the degree of anisotropy currently characterizing the system.

More generally, for clusters at different dynamical stages a radial variation of the SG to FG velocity dispersion could be due to a combination of the effect of binaries and a radial gradient in the fraction of SG stars.

Moving a step forward in our comprehension of the kinematics of MPs is in turn a key stage in the study of GC formation and evolution.

The authors thank the referee Mario Mateo for the careful reading of the paper and the useful comments that improved the presentation of this work. E.D. acknowledges support from The Leverhulme Trust Visiting Professorship Programme VP22017-030.

\section{ORCID iDs}

E. Dalessandro (1) https://orcid.org/0000-0003-4237-4601

A. Mucciarelli (16 https://orcid.org/0000-0001-9158-8580

A. Sollima (1) https://orcid.org/0000-0001-7484-8467

F. R. Ferraro (1) https://orcid.org/0000-0002-2165-8528

R. Ibata (i) https://orcid.org/0000-0002-3292-9709

B. Lanzoni iํ https://orcid.org/0000-0001-5613-4938

D. Massari (i) https://orcid.org/0000-0001-8892-4301

\section{References}

Bastian, N., Lamers, H. J. G. L. M., de Mink, S. E., et al. 2013, MNRAS, 436, 2398

Bastian, N., \& Lardo, C. 2017, arXiv:1712.01286

Bekki, K. 2010, ApJL, 724, L99
Bellazzini, M., Bragaglia, A., Carretta, E., et al. 2012, A\&A, 538, A18 Bellini, A., Piotto, G., Milone, A. P., et al. 2013, ApJ, 765, 32 Bellini, A., Vesperini, E., Piotto, G., et al. 2015, ApJL, 810, L13 Bragaglia, A., Carretta, E., D'Orazi, V., et al. 2017, A\&A, 607, A44

Chung, C., Yoon, S.-J., \& Lee, Y.-W. 2011, ApJL, 740, L45

Cordero, M. J., Hénault-Brunet, V., Pilachowski, C. A., et al. 2017, MNRAS, 465,3515

Dalessandro, E., Cadelano, M., Vesperini, E., et al. 2018, ApJ, 859, 15

Dalessandro, E., Lapenna, E., Mucciarelli, A., et al. 2016, ApJ, 829, 77

Dalessandro, E., Massari, D., Bellazzini, M., et al. 2014, ApJ, 791L, 4

Decressin, T., Meynet, G., Charbonnel, C., Prantzos, N., \& Ekström, S. 2007, A\&A, 464, 1029

de Mink, S. E., Pols, O. R., Langer, N., \& Izzard, R. G. 2009, A\&A, 507, L1

Denissenkov, P. A., \& Hartwick, F. D. A. 2014, MNRAS, 437, 21

D'Ercole, A., D'Antona, F., Ventura, P., Vesperini, E., \& McMillan, S. L. W. 2010, MNRAS, 407, 854

D’Ercole, A., Vesperini, E., D'Antona, F., McMillan, S. L. W., \& Recchi, S. 2008, MNRAS, 391, 825

Duquennoy, A., \& Mayor, M. 1991, A\&A, 248, 485

Foreman-Mackey, D., Hogg, D. W., Lang, D., \& Goodman, J. 2013, PASP, 125,306

Gratton, R. G., Carretta, E., \& Bragaglia, A. 2012, A\&ARv, 20, 50

Heggie, D., \& Hut, P. (ed.) 2003, The Gravitational Million-Body Problem: A Multidisciplinary Approach to Star Cluster Dynamics (Cambridge: Cambridge Univ. Press)

Hénault-Brunet, V., Gieles, M., Agertz, O., \& Read, J. I. 2015, MNRAS, 450,1164

Hong, J., Vesperini, E., Sollima, A., et al. 2015, MNRAS, 449, 629

Hong, J., Vesperini, E., Sollima, A., et al. 2016, MNRAS, 457, 4507

Hut, P., \& Bahcall, J. N. 1983, ApJ, 268, 319

King, I. R. 1966, AJ, 71, 64

Kirby, E. N., Simon, J. D., \& Cohen, J. G. 2015, ApJ, 810, 56

Lardo, C., Bellazzini, M., Pancino, E., et al. 2011, A\&A, 525, A114

Larsen, S. S., Baumgardt, H., Bastian, N., et al. 2015, ApJ, 804, 71

Larsen, S. S., Brodie, J. P., Grundahl, F., \& Strader, J. 2014, ApJ, 797, 15

Lee, H. M., \& Nelson, L. A. 1988, ApJ, 334, 688

Lind, K., Asplund, M., Barklem, P. S., \& Belyaev, A. K. 2011, A\&A, 528, 103

Lucatello, S., Sollima, A., Gratton, R., et al. 2015, A\&A, 584, A52

Martocchia, S., Cabrera-Ziri, I., Lardo, C., et al. 2018, MNRAS, 473, 2688

Massari, D., Lapenna, E., Bragaglia, A., et al. 2016, MNRAS, 458, 4162

Massari, D., Mucciarelli, A., Dalessandro, E., et al. 2017, MNRAS, 468, 1249

Mastrobuono-Battisti, A., \& Perets, H. B. 2013, ApJ, 779, 85

Miholics, M., Webb, J. J., \& Sills, A. 2015, MNRAS, 454, 2166

Milone, A. P., Piotto, G., Bedin, L. R., et al. 2012, ApJ, 744, 58

Mucciarelli, A., Bellazzini, M., Catelan, M., et al. 2013a, MNRAS, 435, 3667

Mucciarelli, A., Bellazzini, M., Ibata, R., et al. 2012, MNRAS, 426, 2889

Mucciarelli, A., Dalessandro, E., Massari, D., et al. 2016, ApJ, 824, 73

Mucciarelli, A., Origlia, L., Ferraro, F. R., \& Pancino, E. 2009, ApJ, 659L, 134

Mucciarelli, A., Pancino, E., Lovisi, L., Ferraro, F. R., \& Lapenna, E. 2013b, ApJ, 766, 78

Muratov, A. L., \& Gnedin, O. Y. 2010, ApJ, 718, 1266

Pasquini, L., Avila, G., Allaert, E., et al. 2000, Proc. SPIE, 4008, 129

Piotto, G., Milone, A. P., Bedin, L. R., et al. 2015, AJ, 149, 91

Plummer, H. C. 1911, MNRAS, 71, 460

Pryor, C., \& Meylan, G. 1993, in ASP Conf. Ser. 50, Structure and Dynamics of Globular Clusters, ed. S. G. Djorgovski \& G. Meylan (San Francisco, CA: ASP), 357

Richer, H. B., Heyl, J., Anderson, J., et al. 2013, ApJL, 771, L15

Savino, A., Massari, D., Bragaglia, A., Dalessandro, E., \& Tolstoy, E. 2018, MNRAS, 474, 4438

Sbordone, L., Salaris, M., Weiss, A., \& Cassisi, S. 2011, A\&A, 534, A9

Schiavon, R. P., Caldwell, N., Conroy, C., et al. 2013, ApJL, 776, L7

Stetson, P. B., \& Pancino, E. 2008, PASP, 120, 1332

van de Ven, G., van den Bosch, R. C. E., Verolme, E. K., \& de Zeeuw, P. T. 2006, A\&A, 445, 513

Vesperini, E., Hong, J., Webb, J. J., D’Antona, F., \& D'Ercole, A. 2018, MNRAS, 476, 2731

Vesperini, E., McMillan, S. L. W., D’Antona, F., \& D'Ercole, A. 2011, MNRAS, 416, 355

Vesperini, E., McMillan, S. L. W., D’Antona, F., \& D'Ercole, A. 2013, MNRAS, 429, 1913

Watkins, L. L., van der Marel, R. P., Bellini, A., \& Anderson, J. 2015, ApJ, 803, 29 